\title{
Predicting lung cancer recurrence from circulating tumour DNA. Commentary on 'Phylogenetic ctDNA analysis depicts early-stage lung cancer evolution'
}

\author{
Daniel J Murphy ${ }^{\star, 1}$ and Kevin G Blyth ${ }^{2,3}$
}

Cell Death and Differentiation (2017) 24, 1473-1474; doi:10.1038/cdd.2017.97; published online 16 June 2017

Cancers of the lung kill more people worldwide than cancers of any other organ. Approximately $16 \%$ of lung cancer cases are suitable for surgical resection; however, fatal disease recurs in some $30-70 \%$ of resected cases. ${ }^{1,2}$ Better predictive indicators of tumour recurrence could lead to improved treatment outcomes in patients with relapse while sparing others from unnecessary procedures. The study by Abbosh et al. ${ }^{3}$ used patient-specific multiplex PCR coupled with next-generation sequencing (NGS) to examine plasma-borne tumour-derived cell-free DNA (alt. circulating tumour DNA, ctDNA) as a predictive biomarker of post-resection tumour recurrence in patients with early stage NSCLC. Conducted under the auspices of the TRACERx clinical trial (TRAcking non-small cell lung cancer evolution through therapy $(\mathrm{Rx})),{ }^{4}$ the study addressed the clinico-pathologic determinants of ctDNA, the clonal/subclonal fidelity of ctDNA, and the potential for ctDNA detection and characterisation not only to predict relapse incidence but, more importantly, to identify potentially druggable biological features of recurrent disease Figure 1.

The presence of cell-free DNA (cfDNA) in plasma has long been appreciated to rise in response to a spectrum of physiological and disease states. ${ }^{5}$ The distinction of $\operatorname{ctDNA}$ depends on detection of tumour-associated mutations in circulating cfDNA, typically in the form of single-nucleotide variants (SNVs). Using a threshold of detection of two SNVs to score a plasma sample positive for ctDNA, Abbosh and colleagues showed clear correlations of ctDNA presence in plasma with primary tumour proliferative index (Ki67); lymphovascular invasion; and degree of tumour necrosis. They also revealed a strong correlation with tumour volume that has implications for detection of minimal or residual disease (see below). Strikingly, the study revealed a preferential association between presence of ctDNA and tumour histological subtype, whereas pre-operative plasma from 30 out of 31 lung squamous cell carcinoma (LuSC) patients scored positive: only 11 out of 58 cases of lung adenocarcinoma (LuAD) scored positive. Importantly, this pattern was preserved in a subgroup comparison of stage-matched (stage I) LuSC and LuAD cases (16 out of 17 (94\%) detected versus 5/39 (13\%) detected, respectively). Furthermore, non-adenocarcinoma histology was the single strongest determinant of ctDNA presence. $^{3}$ This may reflect a greater propensity for LuSC to be micro-metastatic, even at Stage I, but also suggests that ctDNA analysis will have little value for a large proportion of LuAD cases.

The next phase of the study compared longitudinal detection of ctDNA, pre- and post-operatively, with periodic clinical assessment and chest radiography, in a subset of NSCLC samples, including both LuSC and LuAD. CtDNA was detected in 13 of 14 relapse cases, on average some 70 days prior to clinical confirmation by CT imaging. On the other hand, ctDNA was detected in 1 of 10 cases with no evidence of clinical relapse (at the time of publication). In a small number of cases, tracking the number of SNVs post-operatively appeared to reflect resistance or indeed sensitivity to adjuvant chemotherapy, as three patients whose plasma SNV numbers rose while receiving chemotherapy all relapsed within 1 year, while one patient that exhibited a sharp decrease in SNVs remained relapse-free 688 days later. ${ }^{3}$ While these data are certainly encouraging, they suggest that further studies in larger cohorts are warranted.

The methods used to define recurrent disease might also require adjustment in future studies. This would allow clearer definition of the temporal relationship between the emergence of recurrent disease and detection of ctDNA. In TRACERx, a standard UK follow-up schedule was used, involving clinical review (usually limited to discussion of symptoms and clinical examination) combined with a chest radiograph, without any protocolised cross-sectional imaging. ${ }^{2}$ CT scanning was only triggered by new symptoms or a change in chest radiographic appearances. This practice is based on a lack of high-quality evidence that scheduled CT surveillance improves survival ${ }^{6,7}$ despite the previous data showing improved sensitivity for early detection using surveillance $\mathrm{CT}^{8}$ As a result, it is highly likely that tumour recurrence had in fact developed well before the 70 days described between the emergence of ctDNA and 'clinical' detection. For similar reasons, it is difficult to draw conclusions from the report of ctDNA detection in the single patient described 'without clinical evidence of recurrence', given the low sensitivity of the follow-up methods used. The

\footnotetext{
${ }^{1}$ Institute of Cancer Sciences, University of Glasgow, Glasgow, G61 1BD, UK; ${ }^{2}$ Pleural Disease Unit, Queen Elizabeth University Hospital, Glasgow, UK and ${ }^{3}$ Institute of Infection, Immunity and Inflammation, University of Glasgow, Glasgow, UK

*Corresponding author: DJ Murphy, Institute of Cancer Sciences, CRUK Beatson Institute, University of Glasgow, Garscube Estate, Switchback Rd, Glasgow G61 1BD, UK. Tel: +441413308710; E-mail: Daniel.Murphy@glasgow.ac.uk
} 


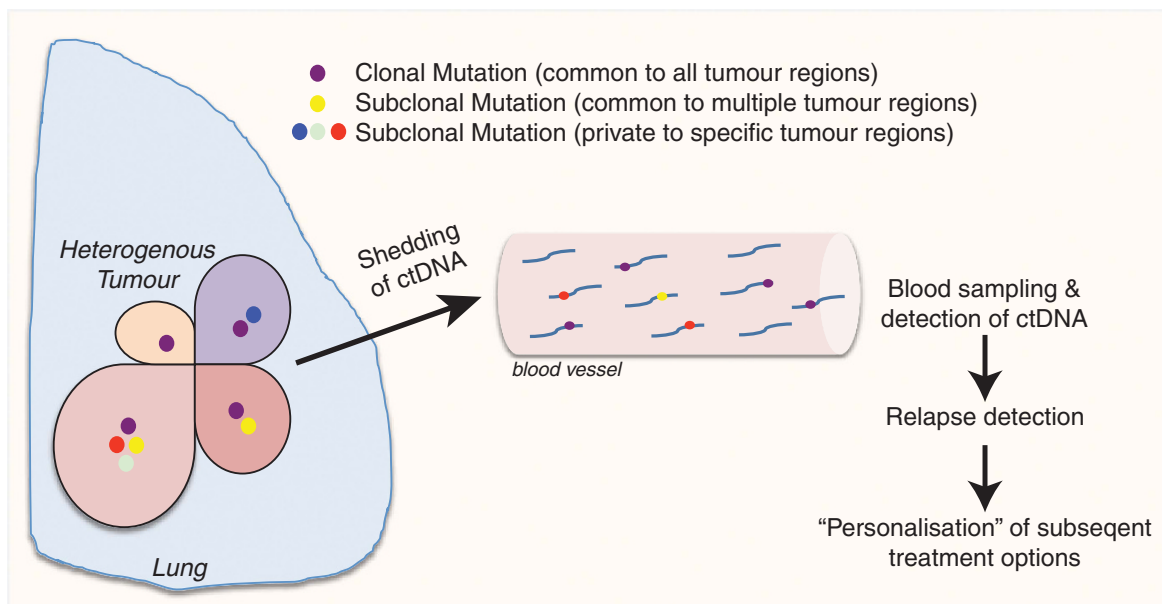

Figure 1 Distinct tumour sub-populations are characterised by the spectrum of mutations present: mutations common to all regions are classed as clonal, whereas those present in a subset of regions are classed as subclonal. Shedding of detectable ctDNA from different tumour regions into circulation is correlated to the volume of the tumour subclone. Clonal mutations are more likely to be detected than subclonal mutations.

authors' description of good correlation between tumour volume at diagnosis and ctDNA variant allele frequency (VAF), and an ability to detect ctDNA shed from tiny primary tumours would tend to support this. Therefore, future studies incorporating US-style lung cancer follow-up, which includes 6-monthly CT scans for 2 years post-surgery, ${ }^{9}$ are likely to better define the relationship between the emergence of recurrent disease and ctDNA, and the true lead-time benefit of this new and exciting technology.

Perhaps the greatest value in the approach derives from analysis of the actual SNVs detected, many of which reflect functional mutations present in the primary tumours. Multiregion NGS of the primary tumours allowed the researchers to assemble phylogenetic 'trees' of each tumour, distinguishing clonal mutations present in all tumour regions from subclonal mutations occurring in a subset of tumour regions. Transposing this analysis onto the detected ctDNA revealed which tumour subclones were represented in ctDNA pre- and postoperatively. Analysis of clonal dynamics during tumour relapse shed light on whether recurring tumours were likely to be mono- or oligo-clonal and, in the former instance, which tumour subpopulation was driving tumour relapse. The power of this approach to potentially guide therapy is exemplified by one particular case, in which ctDNA analysis during relapse indicated re-emergence of clonal disease that mapped back to a particular subclone of the primary tumour containing an ERBB2 amplification. However, for reasons that are unclear, the patient was subsequently administered an EGFRselective inhibitor (Erlotinib), whereas, in our opinion, an
ERBB2-selective or multi-ERBB inhibitor (e.g., Afatinib) would likely have yielded greater benefit. ${ }^{10-12}$

Overall, the study quite firmly establishes the feasibility of longitudinal monitoring of ctDNA as a surrogate for tumour recurrence, at least in the LuSC subtype of NSCLC. The authors quite rightly point out that the approach is presently limited by cost and by the sensitivity of current ctDNA platforms, neither of which are insurmountable in the medium term. The greater challenges moving forward will be to apply this technology meaningfully towards the vast majority of NSCLC cases that are unsuitable for surgical resection and indeed to better define the subgroup of LuAD cases that might benefit from the approach.

\section{Conflict of Interest}

The authors declare no conflict of interest.

1. Pignon JP et al. J Clin Oncol 2008; 26: 3552-3559.

2. NICE Clinical Guidelines. 2011 Update. Available at: https://www.ncbi.nlm.nih.gov/pubmed/ 22855970. Accessed date May 2017.

3. Abbosh $\mathrm{C}$ et al. Nature 2017 (in press).

4. Jamal-Hanjani M et al. PLOS Biol 2014; 12: e1001906.

5. Wan JC et al. Nat Rev Cancer 2017; 17: 223-238.

6. Calman L et al. J Thorac Oncol 2011; 6: 1993-2004.

7. Crabtree TD et al. J Thorac Cardiovasc Surg 2015; 149: 45-52.

8. Hanna WC et al. J Thorac Cardiovasc Surg 2014; 147: 30-33.

9. Colt HG et al. Chest 2013; 143(Suppl 5): e437S-e4354.

10. Suzawa K et al. Cancer Sci 2016; 107: 45-52.

11. Schuler M et al. Ann Oncol 2016; 27: 417-423.

12. Mazieres J et al. Ann Oncol 2016; 27: 281-286. 